\title{
Post-Procedure Mortality after Cardiovascular Treatment Procedures in Patients with Pneumoconiosis
}

\author{
Alexander V. Sergeev \\ Department of Social and Public Health, Ohio University, Athens, Ohio, USA \\ Email: sergeev@ohio.edu
}

Received 29 November 2014; accepted 17 December 2014; published 13 February 2015

Copyright (C) 2015 by author and Scientific Research Publishing Inc.

This work is licensed under the Creative Commons Attribution International License (CC BY).

http://creativecommons.org/licenses/by/4.0/

(c) (i) Open Access

\section{Abstract}

Pneumoconiosis (PNC) is a major occupational disease that develops as a result of occupational exposure to dust via inhalation. In addition to its harmful effects on the respiratory system, PNC can increase vulnerability to coronary heart disease (CHD) - the leading cause of death in the United States and in the world. Currently, two types of cardiovascular intervention procedures for CHD treatment are percutaneous coronary intervention (PCI) and coronary artery bypass grafting (CABG). The objective of this study was to investigate comparative effectiveness of the two major cardiovascular intervention procedures-PCI and CABG-in PNC patients with CHD. Data from 1094 hospitalizations of adult patients with PNC and CHD (CHD-PNC patients) and 534,120 hospitalizations of CHD patients without PNC (CHD-nonPNC) were investigated. Adjusted odds ratios for in-hospital death in relation to the type of procedure, adjusted for patient socio-demographic and clinical characteristics and hospital characteristics, were calculated using multivariable logistic regression. Men constituted $97.8 \%$ of CHD-PNC patients and $68.6 \%$ of CHD-nonPNC patients. Within the CHD-PNC group, crude (unadjusted) in-hospital mortality after CABG and PCI did not differ significantly $(1.35 \%$ vs. $2.00 \%, p=0.425)$ and remained insignificant in the multivariable analysis, adjusted for patient and hospital characteristics (adjusted OR $=0.714,95 \%$ CI $0.220-2.323$, $\mathrm{p}=\mathbf{0 . 5 7 6}$ ). But in the CHD-nonPNC group, in-hospital mortality after CABG was significantly higher than after PCI both in crude analysis $(2.83 \%$ vs. $1.28 \%, \mathrm{p}<0.001)$ and adjusted analysis (adjusted $O R=1.637,95 \%$ CI $1.541-1.738, p<0.001)$. The study results indicate that CABG is as safe as PCI in male CHD-PNC patients, in terms of in-hospital mortality. Further studies investigating comparative effectiveness of cardiovascular procedures in PNC patients using more detailed data are warranted.

\section{Keywords}

Occupational Lung Disease, Pneumoconiosis, Complications, Cardiovascular Disease, Cardiovascular Treatment Procedures, Mortality 


\section{Introduction}

Pneumoconiosis is a major occupational disease that develops as a result of occupational exposure to dust via inhalation. Pneumoconiosis was responsible for more than 26,000 deaths in the US during 2000-2010 [1]. There are several types of pneumoconiosis. Although strict medical definitions suggest that only interstitial lung diseases caused by inorganic dust belong to this group (such as coal dust, silica, asbestos, aluminum, beryllium, talc) [2], other authorities extend the definition of pneumoconiosis to include organic dusts, such as byssinosis which is caused by cotton dust [1]. Most pneumoconioses share some common pathophysiological mechanisms that include excessive development of fibrous (i.e., scar) tissue in the lungs—pneumofibrosis-that restricts respiratory lung capacity. What makes pneumoconiosis a particularly dangerous disease is that a) once pneumofibrosis is initiated, it may continue progressing even after the dust exposure is discontinued; and b) no effective treatment for pneumoconiosisis currently available [2] [3]. Prevention and reduction of work-related interstitial lung diseases is a strategic goal of the National Institute's for Occupational Safety and Health (NIOSH) Respiratory Disease Research Program [4].

In addition to its harmful effect on respiratory system, pneumoconiosis has been shown to be an emerging risk factor for coronary heart disease (CHD) — the leading cause of death in the US and in the world [5] [6]. A study of over 9000 underground coal miners found a dose-response relationship between cumulative coal dust exposure and CHD mortality [7]. A statistically significant increase in CHD mortality was also found in a study of 12,000 silica-exposed workers followed-up for 25 years [8]. CHD morbidity and mortality in coal-mining areas of Appalachia are higher than in non-coal mining areas of the region [9] [10]. CHD is a disease that causes myocardial ischemia (i.e., lack of oxygenated blood supply to the heart muscle) due to coronary atherosclerosis (hardening of the heart arteries) and hypercoagulation (i.e., prone to blood clotting) states. The major mechanism responsible for the cardiovascular effect of inhaled particles is a low-grade inflammation in the lung tissue surrounding the particles. Inflammation increases plasma levels of coagulants, such as fibrinogen [11] [12], and previous research has demonstrated that plasma levels of fibrinogen are higher in coal miners with pneumoconiosis than without pneumoconiosis [13].

Currently, two types of cardiovascular intervention procedures for CHD treatment are percutaneous coronary intervention (PCI) and coronary artery bypass grafting (CABG). The PCI procedure consists of stretching the coronary stenosis by inflating a small balloon guided through a catheter and placing a stent (i.e., a small mesh tube) in the artery to prevent re-stenosis. The CABG is a more invasive and high-risk procedure that includes taking a blood vessel from another part of the body and grafting it to the patient's coronary artery to bypass the stenosis. In some cases, the choice of which procedure to use is based on other medical indications, but in many cases, the choice of procedure is not clearly indicated by extant evidence. The federal Agency for Healthcare Research and Quality (AHRQ) points out that comparative effectiveness of PCI and CABG in patients with coexisting (comorbid) conditions remains an open question and needs to be investigated [14].

\section{Research Methods and Study Design}

\subsection{Study Population}

Subject selection was based on the following inclusion criteria: adult patients (21 years and older) with CHD who were admitted to the hospital and underwent either PCI or CABG. Patient data from hospitalizations of adults 21 years and older who underwent either a PCI or CABG procedure were obtained from the State Inpatient Databases (SID) developed within the scope of a Federal-State-Industry partnership [15]. The SID were developed as a part of a larger Healthcare Cost and Utilization Project (HCUP), sponsored by the AHRQ. Both high validity and generalizability are the major strengths of the SID databases because they contain the universe of hospitalized patients' data from hospital charts in participating states. To protect patients' confidentiality, the SID data are de-identified. The SID contain detailed information on patients' clinical characteristics (including the primary and secondary diagnoses; co-existing conditions; diagnostic and treatment procedures; information on admittance and discharge/disposition of patient; length of stay etc.), demographics (age, gender, race/ethnicity), non-identifying residential (rural-urban) and socio-economic status (median household income by patient's zip code of residence); information on hospital charges and payment, etc., for a total of more than 200 data elements (some elements are not available in all the participating states' databases). The SID data are coded uniformly throughout the databases. Information on patients’ Diagnosis-Related Group (DRG) and ICD-9-CM 
(International Classification of Diseases, the $9^{\text {th }}$ Revision, Clinical Modification) codes for diagnoses and both diagnostic and treatment procedures are available for all participating states. Subjects were selected from the following 13 states: Arizona, California, Colorado, Florida, Kentucky, Maryland, Massachusetts, Rhode Island, New Jersey, New York, Utah, Washington, and West Virginia for 2007 and 2009. This methodological approach ensured the generalizability of the study results, because the 13 states listed above constitute about $38 \%$ of the total country population for those years [16].

The primary outcome of interest was in-hospital mortality. Patient demographic characteristics, socio-economic status and clinical characteristics as well as hospital data were used to control for confounding. Income data (median household income state quartile for patient's zip code of residence) were used as a proxy measure of socio-economic status.

Because presence of other, co-existing conditions (comorbidities) in pneumoconiosis patients with CHD can negatively affect their mortality we utilized AHRQ's Elixhauser Comorbidity Measures that were specifically developed for use with large patient databases [17]. Elixhauser Comorbidity Measures are comprised of 29 major comorbidity categories (groups). To achieve effective adjustment for both the number and severity of comorbidities without compromising the parsimony of the models, we utilized Elixhauser-Walraven scores summarizing all the Elixhauser comorbidities into a single index for each patient [18]. Because by definition, all the pneumoconiosis patients have a chronic pulmonary (respiratory) disorder, we modified the Elixhauser-Walraven score to exclude the chronic pulmonary disease category.

Patient emergency admission status was identified by the SID Emergency Department Service indicator.

\subsection{Statistical Analyses}

Because hospital length of stay after the procedure is relatively short (days, not months or years), survival vs. death status (i.e., in-hospital mortality) was defined as a binary/dichotomous variable: discharged alive (i.e., survived) vs. deceased (i.e., did not survive). In preliminary unadjusted analyses, in-hospital mortality and patients' comorbidity scores were compared between CABG and PCI groups using chi-square test (for mortality as a dichotomous variable) and t-test (for comorbidity scores as a continuous variable).

In adjusted analyses, multivariable statistical methods were used to control (adjust for) known confounders/ covariates. Because of a binary/dichotomous nature of in-hospital mortality as the dependent variable and a relatively short length of stay, multivariable logistic regression was used for adjusted analysis of mortality, rather than Kaplan-Meier survival curves or the Cox proportional hazards model that would require a substantially longer observation time. Both 95\% confidence intervals (95\% CI) and respective p-values were obtained for each of the beta coefficient estimates of the multivariable model to determine which continuous and categorical predictors were related to the dependent variable.

Statistical analyses were performed using SAS software, version 9.2 (SAS Institute Inc., Cary, NC). For statistical significance testing, the conventional level of type I error (alpha-level) of 0.05 was used in all data analyses $(\mathrm{p}<0.05)$.

\section{Results}

\subsection{Patient Characteristics}

We identified a total of 535,214 hospitalizations of CHD patients 21 years of age and older who underwent either CABG or PCI. Of them, there were 1094 hospitalizations of patients with pneumoconiosis and CHD (CHDPNC patients) and 534,120 hospitalizations of CHD patients without pneumoconiosis (CHD-nonPNC).

Men constituted $97.8 \%$ of CHD-PNC patients (1070 patients), and women-2.2\% (24 patients), which is consistent with mostly men being employed in pneumoconiosis-associated industries, such as mining. Of the $24 \mathrm{fe}-$ male patients, CABG was performed in 12 patients and PCI was performed in 12 other patients. There were no deaths in CHD-PNC female patients. Due to their extremely small numbers that can compromise the parsimony of the statistical models and because gender is a known confounder in studies of CHD, we utilized restriction as an established method of confounding control and did not include women in further multivariable analyses. Instead, we conducted a separate subgroup analysis for the 24 women; it is presented in the Unadjusted Analysis section below.

Among CHD-nonPNC patients, men constituted 68.6\% (366,267 patients), and women-31.4\% (167,853 pa- 
tients).

The mean \pm standard deviation (SD) age of the CHD-PNC patients was $71.8 \pm 9.05$ years, and of the CHDnonPNC patients-65.6 \pm 11.9 years.

\subsection{Unadjusted Analysis}

In crude (unadjusted) analysis, in-hospital mortality in CHD-PNC patients was similar after CABG and PCI $(1.35 \%$ vs. $2.00 \%, p=0.425)$. However, in CHD-nonPNC patients, unadjusted in-hospital mortality was significantly higher after CABG than after PCI (2.83\% vs. $1.28 \%$, $\mathrm{p}<0.001)$.

Both Elixhauser-Walraven comorbidity original and modified scores were higher in CHD-PNC patients as compared to CHD-nonPNC patients (5.06 \pm 4.63 vs. $1.92 \pm 4.57, \mathrm{p}<0.001$; and, $2.07 \pm 4.64$ vs. $1.47 \pm 4.32$, $\mathrm{p}<$ 0.001 , respectively), with higher scores being indicative of more severe co-existing conditions (comorbidities).

\subsection{Adjusted Analysis}

Adjusted analysis was conducted using multivariable logistic regression allowing to control/adjust for confounders/covariates - patient and hospital characteristics. Results of the adjusted analysis of in-hospital mortality in CHD-PNC males (Table 1) demonstrated that after controlling for the effect of age, race, income, comorbidity, rurality, and emergency department use status, in-hospital mortality did not differ in CABG and PCI patients (adjusted OR $=0.714,95 \%$ CI $0.220-2.323$, p = 0.576).

However, in CHD-nonPNC males adjusted in-hospital mortality was statistically significantly higher in patients who underwent CABG compared to those who underwent PCI (adjusted OR $=1.637,95 \%$ CI 1.541 1.738, $\mathrm{p}<0.001$; Table 2). Also, as expected, both in CHD-PNC and CHD-nonPNC groups, patients of older

Table 1. Adjusted analysis of in-hospital mortality in CHD-PNC male patients.

\begin{tabular}{|c|c|c|c|c|}
\hline Characteristic & $\begin{array}{l}\beta \text { coefficient } \\
\text { estimate }\end{array}$ & Standard error & OR (95\% CI) & p-value \\
\hline CABG (compared to PCI) ${ }^{a}$ & -0.3364 & 0.6018 & $0.714(0.220,2.323)$ & 0.576 \\
\hline Age, years ${ }^{b}$ & 0.0615 & 0.0348 & $1.063(0.993,1.138)$ & 0.077 \\
\hline \multicolumn{5}{|l|}{ Race (compared to Caucasians) ${ }^{c}$} \\
\hline African Americans & 1.0977 & 1.3375 & $2.997(0.218,41.227)$ & 0.411 \\
\hline Other & -0.0576 & 0.7109 & $0.944(0.234,3.803)$ & 0.935 \\
\hline \multicolumn{5}{|l|}{$\begin{array}{l}\text { Median household income state quartile for } \\
\text { patient zip code (compared to Q4) }\end{array}$} \\
\hline Q1 & -0.1827 & 0.9315 & $0.833(0.134,5.171)$ & 0.844 \\
\hline Q2 & -0.1461 & 0.9300 & $0.864(0.140,5.348)$ & 0.875 \\
\hline Q3 & -0.3979 & 0.9725 & $0.672(0.100,4.519)$ & 0.682 \\
\hline $\begin{array}{l}\text { Modified Elixhasuer-Walraven } \\
\text { comorbidity score }^{e}\end{array}$ & 0.1644 & 0.0377 & $1.179(1.095,1.269)$ & $<0.001$ \\
\hline \multicolumn{5}{|l|}{ Rurality (compared to isolated rural area) ${ }^{f}$} \\
\hline Urban & -0.8328 & 0.9305 & $0.435(0.070,2.694)$ & 0.370 \\
\hline Large rural town & -0.1814 & 1.1327 & $0.834(0.091,7.679)$ & 0.872 \\
\hline Small rural town & 0.9667 & 0.9963 & $2.629(0.373,18.532)$ & 0.332 \\
\hline Emergency Department admission ${ }^{g}$ & 0.5977 & 0.5721 & $1.818(0.592,5.578)$ & 0.296 \\
\hline
\end{tabular}

${ }^{a}$ Adjusted for age, race, median household income, comorbidity, rurality, emergency department admittance; ${ }^{b}$ adjusted for the type of procedure, race, median household income, comorbidity, rurality, emergency department admittance; ' adjusted for the type of procedure, age, median household income, comorbidity, rurality, emergency department admittance; ${ }^{d}$ adjusted for the type of procedure, age, race, comorbidity, rurality, emergency department admittance; ${ }^{e}$ adjusted for the type of procedure, age, race, median household income, rurality, emergency department admittance; ${ }^{\text {adjusted }}$ for the type of procedure, age, race, median household income, comorbidity, emergency department admittance; ${ }^{g}$ adjusted for the type of procedure, age, race, median household income, comorbidity, rurality. 
Table 2. Adjusted analysis of in-hospital mortality in CHD-nonPNC male patients.

\begin{tabular}{|c|c|c|c|c|}
\hline Characteristic & $\beta$ coefficient estimate & Standard error & OR (95\% CI) & p-value \\
\hline CABG (compared to PCI) ${ }^{a}$ & 0.4926 & 0.0307 & $1.637(1.541,1.738)$ & $<0.001$ \\
\hline Age, years ${ }^{b}$ & 0.0351 & 0.0013 & $1.036(1.033,1.039)$ & $<0.001$ \\
\hline \multicolumn{5}{|l|}{ Race (compared to Caucasians) ${ }^{c}$} \\
\hline African Americans & -0.1279 & 0.0724 & $0.880(0.763,1.014)$ & 0.077 \\
\hline Other & 0.1563 & 0.0341 & $1.169(1.094,1.250)$ & $<0.001$ \\
\hline \multicolumn{5}{|l|}{$\begin{array}{l}\text { Median household income state quartile for } \\
\text { patient zip code (compared to Q4) }\end{array}$} \\
\hline Q1 & 0.1765 & 0.0439 & $1.193(1.095,1.300)$ & $<0.001$ \\
\hline Q2 & 0.0986 & 0.0426 & $1.104(1.015,1.200)$ & 0.021 \\
\hline Q3 & 0.0383 & 0.043 & $1.039(0.955,1.131)$ & 0.373 \\
\hline $\begin{array}{l}\text { Modified Elixhasuer-Walraven } \\
\text { comorbidity score }^{e}\end{array}$ & 0.141 & 0.0021 & $1.151(1.147,1.156)$ & $<0.001$ \\
\hline \multicolumn{5}{|l|}{ Rurality (compared to isolated rural area) ${ }^{f}$} \\
\hline Urban & -0.1495 & 0.0961 & $0.861(0.713,1.040)$ & 0.120 \\
\hline Large rural town & -0.0039 & 0.1089 & $0.996(0.805,1.233)$ & 0.972 \\
\hline Small rural town & -0.1454 & 0.1262 & $0.865(0.675,1.107)$ & 0.250 \\
\hline Emergency Department admission ${ }^{g}$ & 0.7358 & 0.0302 & $2.087(1.967,2.214)$ & $<0.001$ \\
\hline
\end{tabular}

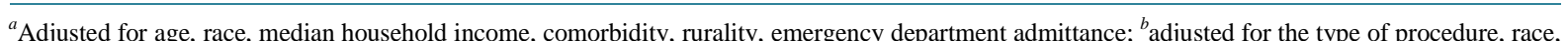
median household income, comorbidity, rurality, emergency department admittance; ${ }^{c}$ adjusted for the type of procedure, age, median household income, comorbidity, rurality, emergency department admittance; ${ }^{d}$ adjusted for the type of procedure, age, race, comorbidity, rurality, emergency department admittance; ${ }^{e}$ adjusted for the type of procedure, age, race, median household income, rurality, emergency department admittance; ${ }^{f}$ adjusted for the type of procedure, age, race, median household income, comorbidity, emergency department admittance; ${ }^{g}$ adjusted for the type of procedure, age, race, median household income, comorbidity, rurality.

age and with more severe comorbidities had higher mortality, regardless of the type of procedure-CABG or PCI. In CHD-nonPNC male patients (but not in CHD-PNC male patients), emergency department status was also statistically significantly associated with in-hospital mortality: higher mortality among patients admitted through emergency department.

\section{Discussion}

The present study of comparative effectiveness of the two major cardiovascular procedures-PCI and CABG - has demonstrated that in PNC patients with CHD, CABG is as safe as PCI in terms of in-hospital mortality.

PNC is a major occupational disease that develops as a result of occupational exposure to dust via inhalation. In addition to its harmful effects on the respiratory system, PNC can increase vulnerability to CHD-the leading cause of death in the US and in the world. Currently, PCI and CABG are the two major cardiovascular intervention procedures currently available for CHD treatment. The AHRQ points out that comparative effectiveness of PCI and CABG in patients with coexisting (comorbid) conditions remains an open question and needs to be investigated [14]. To the best of our knowledge, the present study represents the first comparative effectiveness study of PCI and CABG in patients with PNC.

One of the strengths of this study is strong generalizability if its results. Study subjects were selected from 13 states that constitute about $38 \%$ of the total US population. We used the SID databases that contain the universe of hospitalized patients' data from hospital charts in these states.

Another major strength of the current study is utilization of the established procedures of adjustment/control for the known major confounders of concern in studies of the effectiveness of treatment-demographic and clinical characteristics of study subjects. Lack of control for confounding can make a truly effective treatment to 
wrongly appear as being ineffective or, even worse, an unsafe and/or ineffective procedure to wrongly appear as being safe and effective. Because it is mostly men who are employed in the industries associated with occupational exposure to dust and PNC, there were very few women among study subjects. This is also consistent with a well-established medical knowledge of male gender being a risk factor for atherosclerosis-related diseases, including CHD. Thus, to control for confounding by gender, we applied the method of restriction and included only men in the multivariable analysis. This approach of not including a very small-size subgroup into multivariable models also helped with preserving the parsimony of these models. Using multivariable modeling, we effectively controlled for other major confounders-socio-demographic and clinical characteristics-by including the respective variables as predictors (independent variables) in the models.

Effective control for patients' comorbidities (other co-existing conditions) also strengthened the design of this study. Patients with higher number of co-existing conditions and more severe comorbidities are at higher risk of in-hospital death and also tend to have longer length of stay and higher hospitalization costs. We controlled for patients comorbidity status by quantifying comorbidity with the Elixhauser Comorbidity Measures supplemented with Walraven's scores of severity of the Elixhauser Comorbidity Measures. Because chronic pulmonary disorder is one of the Elixhauser Comorbidity Measures and all PNC patients—by definition-have this disorder, we modified the Elixhauser-Walraven score to exclude the chronic pulmonary disease category.

Adjusted analysis demonstrated that higher Elixhauser-Walraven scores, indicative of more severe comorbidities, are associated with a statistically significant increase in in-hospital mortality. While investigation of this association was not a primary goal of our study, it is consistent with well-established, common medical knowledge and thus serves as an indirect indicator of the quality of our models proving their biological plausibility.

Our study is not free from limitations. This is an observational retrospective cohort study, not a randomized clinical trial, and results from only one observational study are not sufficient to draw an ultimate cause-effect conclusion regarding effectiveness of PCI vs. CABG procedures in patients with PNC. More detailed information on the coronary (heart) arteries affected and the degree and location of stenosis would be helpful, as these factors can influence medical decision-making of the choice of procedure. It is also not impossible that there may be some unaccounted PNC patients who have already developed PNC, but have not had the diagnosis of PNC confirmed officially in their medical records yet, thus appearing in the hospital charts and SID databases as patients without PNC.

In conclusion, the present study has demonstrated that CABG is as safe as PCI in male CHD-PNC patients, in terms of in-hospital mortality. Adjustment for patient socio-demographic and clinical characteristics, including comorbidities, plays an important role in studies of comparative effectiveness of cardiovascular intervention procedures and utilization of healthcare services by PNC patients. Further studies investigating comparative effectiveness of cardiovascular procedures in PNC patients using more detailed data are warranted.

\section{Acknowledgements}

This study was supported by the National Institute for Occupational Safety and Health Pilot Research Project Program of the University of Cincinnati Education and Research Center Grant \#T42/OH008432-06.

\section{References}

[1] National Institute for Occupational Safety and Health (2014) Occupational Respiratory Disease Surveillance: National Occupational Respiratory Mortality System (NORMS). Centers for Disease Control and Prevention (CDC). http://webappa.cdc.gov/ords/norms.html

[2] Weinberger, S. (1998) Principles of Pulmonary Medicine. 3rd Edition, W.B. Saunders Company, Philadelphia.

[3] Bordow, R., Ries, A. and Morris, T., Eds. (2001) Manual of Clinical Problems in Pulmonary Medicine. 5th Edition, Lippincott Williams \& Wilkins, Philadelphia.

[4] National Research Council and Institute of Medicine (2008) Respiratory Disease Research at NIOSH: Reviews of Research Programs of the Institute for Occupational Safety and Health. The National Academies Press, Washington DC.

[5] Go, A.S., Mozaffarian, D., Roger, V.L., Benjamin, E.J., Berry, J.D. and Borden, W.B. (2013) Heart Disease and Stroke Statistics-2013 Update: A Report from the American Heart Association. Circulation, 127, e6-e245. http://dx.doi.org/10.1161/CIR.0b013e318282ab8f

[6] World Health Organization (2008) The Global Burden of Disease: 2004 Update. WHO Press, Geneva.

[7] Landen, D.D., Wassell, J.T., McWilliams, L. and Patel, A. (2011) Coal Dust Exposure and Mortality from Ischemic 
Heart Disease among a Cohort of U.S. Coal Miners. American Journal of Industrial Medicine, 54, 727-733. http://dx.doi.org/10.1002/ajim.20986

[8] Weiner, J., Barlow, L. and Sjogren, B. (2007) Ischemic Heart Disease Mortality among Miners and Other Potentially Silica-Exposed Workers. American Journal of Industrial Medicine, 50, 403-408. http://dx.doi.org/10.1002/ajim.20466

[9] Hendryx, M. (2009) Mortality from Heart, Respiratory, and Kidney Disease in Coal Mining Areas of Appalachia. International Archives of Occupational and Environmental Health, 82, 243-249. http://dx.doi.org/10.1007/s00420-008-0328-y

[10] Hendryx, M. and Zullig, K.J. (2009) Higher Coronary Heart Disease and Heart Attack Morbidity in Appalachian Coal Mining Regions. Preventive Medicine, 49, 355-359. http://dx.doi.org/10.1016/j.ypmed.2009.09.011

[11] Brook, R.D., Franklin, B., Cascio, W., Hong, Y., Howard, G., Lipsett, M., Luepker, R., Mittleman, M., Samet, J., Smith Jr., S.C. and Tager, I. (2004) Air Pollution and Cardiovascular Disease: A Statement for Healthcare Professionals from the Expert Panel on Population and Prevention Science of the American Heart Association. Circulation, 109, 2655-2671. http://dx.doi.org/10.1161/01.CIR.0000128587.30041.C8

[12] Sjogren, B. (1997) Occupational Exposure to Dust: Inflammation and Ischaemic Heart Disease. Occupational and Environmental Medicine, 54, 466-469. http://dx.doi.org/10.1136/oem.54.7.466

[13] Fernandez Rego, G., Ocio Achaerandio, G., Gonzalez Cuervo, V., Rodriquez Menendez, C., Martinez Gonezalez, C. and Alvarez Alvarez, C. (1991) Presence of Acute Phase Response in Coal Workers' Pneumoconiosis. British Journal of Industrial Medicine, 48, 193-195.

[14] Agency for Healthcare Research and Quality (2007) Comparative Effectiveness of Percutaneous Coronary Interventions and Coronary Artery Bypass Grafting for Coronary Artery Disease. DHHS (AHRQ) Publication No. 08-EHC002EF. http://www.effectivehealthcare.ahrq.gov/ehc/products/15/55/CER PCI CABGMainReport.pdf

[15] Agency for Healthcare Research and Quality (2011) Comorbidity Software. http://www.hcup-us.ahrq.gov/toolssoftware/comorbidity/comorbidity.jsp

[16] US Census Bureau (2010) The United States Census Bureau. http://www.census.gov/

[17] Agency for Healthcare Research and Quality (2011) State Inpatient Databases (SID). http://www.hcup-us.ahrq.gov/sidoverview.jsp

[18] van Walraven, C., Austin, P.C., Jennings, A., Quan, H. and Forster, A.J. (2009) A Modification of the Elixhauser Comorbidity Measures into a Point System for Hospital Death Using Administrative Data. Medical Care, 47, 626-633. http://dx.doi.org/10.1097/MLR.0b013e31819432e5 
Scientific Research Publishing (SCIRP) is one of the largest Open Access journal publishers. It is currently publishing more than 200 open access, online, peer-reviewed journals covering a wide range of academic disciplines. SCIRP serves the worldwide academic communities and contributes to the progress and application of science with its publication.

Other selected journals from SCIRP are listed as below. Submit your manuscript to us via either submit@scirp.org or Online Submission Portal.
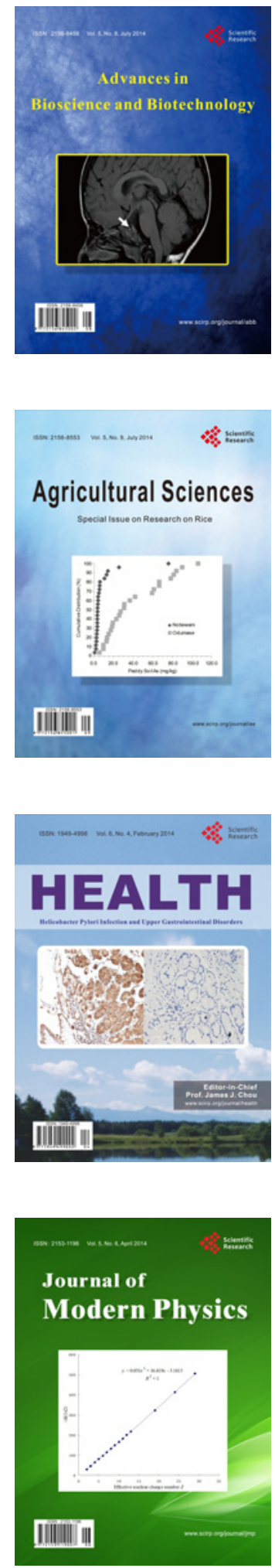
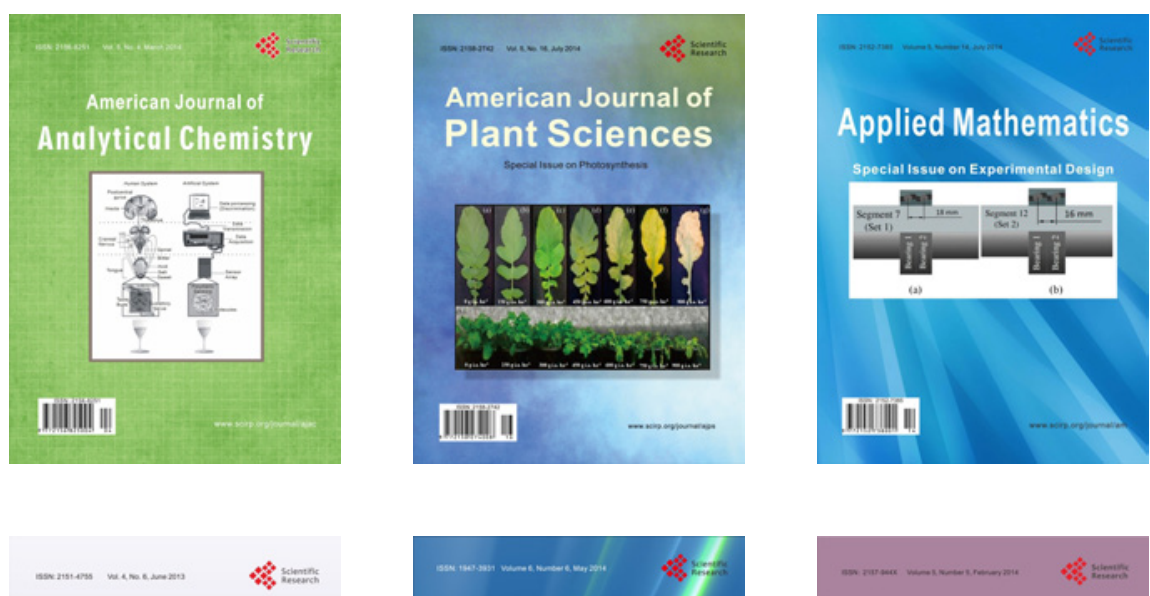

Creative Education
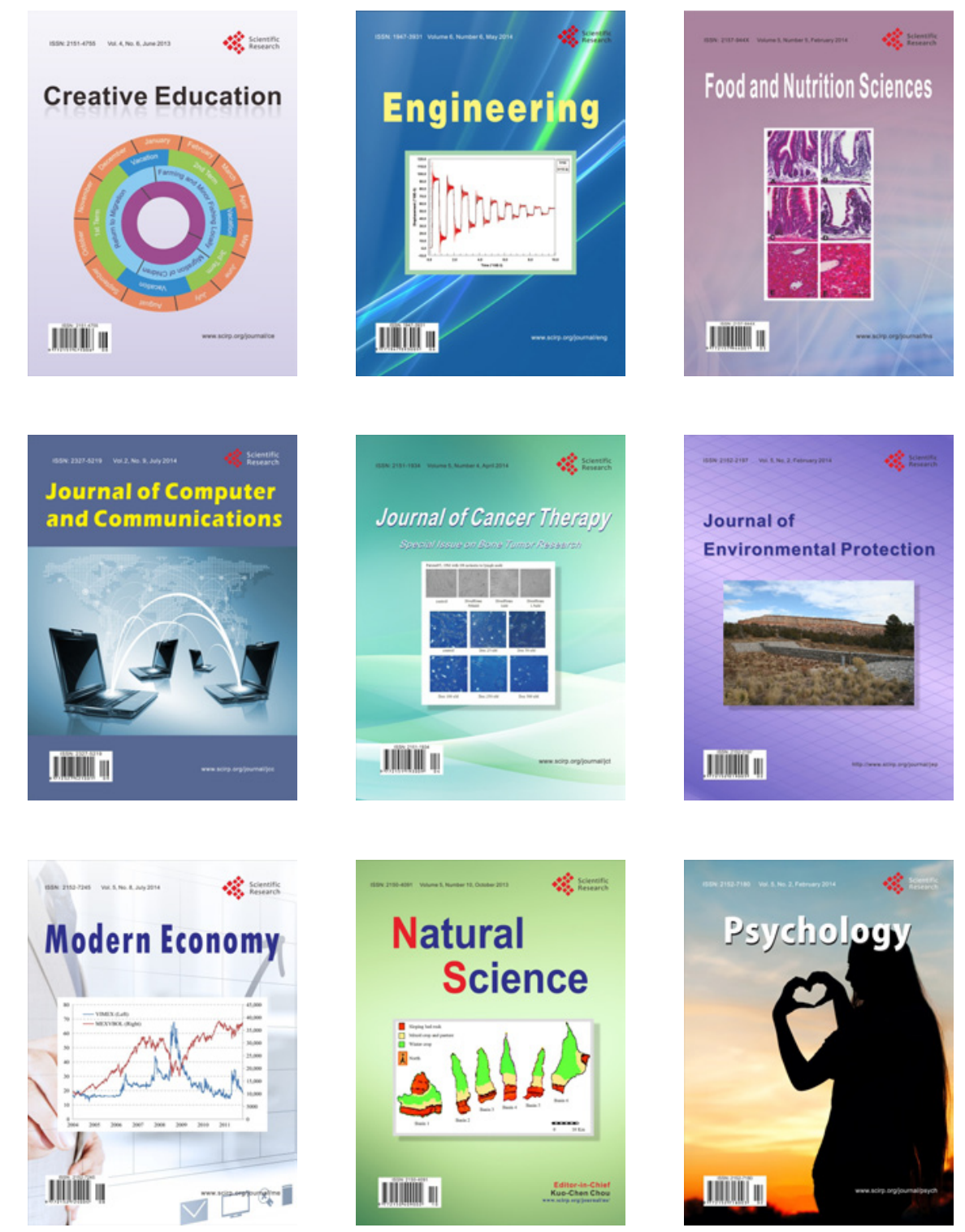